\title{
COINCIDENCE SETS AND TRANSFORMATIONS OF FUNCTION SPACES $\left({ }^{1}\right)$
}

\author{
BY \\ JAMES V. WHITTAKER
}

1. Introduction. When a transformation from a function space $C(X)$ to another $C(Y)$ is given, there is also set up a relation between the subsets of $X$ and those of $Y$ due to the fact that, when two functions agree on a subset of $X$, their images will agree on some subset of $Y$. Three mappings of the subsets of $Y$ into those of $X$ corresponding to a transformation $T$ of $C(X)$ into $C(Y)$ are singled out in this paper, and the first two are studied successively for arbitrary $T$, when $T$ is linear, and when its range is finite dimensional. The third mapping is then introduced, and convergence criteria are obtained for power series in certain classes of transformations. Finally, these results are used to obtain a representation of certain continuous transformations of $C(X)$ onto $C(Y)$ in terms of homeomorphisms of $Y$ into $X$.

2. Coincidence of functions. Let $X$ be a topological space and $C(X)$ the set of continuous, real-valued functions on $X$. If $Y$ is a topological space and $T$ a single-valued transformation of $C(X)$ into $C(Y)$, we define two mappings $\mu_{T}$ and $\pi_{T}$ of $2^{Y}$ into $2^{X}$ : for $D \subset Y$,

$$
\begin{aligned}
& \boldsymbol{\mu}_{\boldsymbol{T}}(D)=\bigcup\{A \subset X: \forall f, g \in C(X), T f=T g|D \Rightarrow f=g| A\}, \\
& \boldsymbol{\pi}_{\boldsymbol{T}}(D)=\cap\{\bar{A} \subset X: \forall f, g \in C(X), f=g|A \Rightarrow T f=T g| D\},
\end{aligned}
$$

where $f \mid A$ is the restriction of $f$ to $A, f=g \mid A$ is short for $f|A=g| A$, and $\bar{A}$ is the closure of $A$. The families of sets on the right sides of (1) and (2) are not empty, for the empty set $\varnothing$ is one of the $A$ 's in (1), and $X$ is one of the 'A's in (2). Evidently $T f=T g \mid D$ implies $f=g \mid \mu_{T}(D)$, and since $\mu_{T}(D)$ is maximal with respect to this property, it, as well as $\pi_{T}(D)$, is closed. Clearly $\mu_{T}(D)$ $=\mu_{T}(\bar{D})$ and $\pi_{T}(D)=\pi_{T}(\bar{D})$. A closed subset $A$ of $X$ will be called a $\pi$-set of $D$ relative to $T$ if, for all $f, g \in C(X), f=g \mid A$ implies $T f=T g \mid D$. Thus $\pi_{T}(D)$ is the intersection of the $\pi$-sets of $D$. A few simple properties of $\mu_{T}$ and $\pi_{T}$ may be noted: $\varnothing$ is a $\pi$-set of $\varnothing$, and $\mu_{T}(\varnothing)=\pi_{T}(\varnothing)=\varnothing$; if $T$ is constant and $D \subset Y$, then $\mu_{T}(D)=\pi_{T}(D)=\varnothing$; if $\varnothing$ is a $\pi$-set of $Y$, then $T$ is constant.

For any $f \in C(X)$, we set $Z(f)=\{x \in X: f(x)=0\}$ and note that $Z(f)$ is closed. Pointwise addition, subtraction, and multiplication of $f$ and $g$ will be denoted by $f+g, f-g$, and $f g$, respectively. We observe that $f=g \mid Z(f-g)$. The constant functions with values 0 and 1 will be denoted by $\theta$ and $u$, respectively.

Received by the editors March 20, 1961.

(1) The author is indebted to the referee for many improvements in the presentation of this paper. 
Lemma 1. If $D$ is a subset of $Y$, then

$$
\mu_{T}(D)=\cap\{Z(f-g): T f=T g \mid D\} .
$$

If $D_{1}, D_{2}$ are subsets of $Y$, and $D_{1} \subset D_{2}$, then

$$
\mu_{T}\left(D_{1}\right) \subset \mu_{T}\left(D_{2}\right), \quad \pi_{T}\left(D_{1}\right) \subset \pi_{T}\left(D_{2}\right) .
$$

If $X$ is completely regular, and $D \subset Y$, then

$$
\mu_{T}(D) \subset \pi_{T}(D) .
$$

If $X$ is normal, and $D \subset Y$, then the family of $\pi$-sets of $D$ relative to $T$ is closed under finite intersections.

Proof. For any $f, g \in C(X)$ satisfying $T f=T g \mid D$, we have $\mu_{T}(D) \subset Z(f-g)$. On the other hand, the right side of (3) is one of the $A$ 's in (1), and (3) is verified. The inclusions in (4) follow immediately from (3) and (2). If $A$ is a $\pi$-set of $D \subset Y$ relative to $T$, and $f=g \mid A$, then $T f=T g \mid D$, and $f=g \mid \mu_{T}(D)$. Thus $Z(f) \supset A$ implies $Z(f) \supset \mu_{T}(D)$, and if $X$ is completely regular, we have $A \supset \mu_{T}(D)$, which establishes (5). Suppose $X$ is normal, $A$ and $B$ are $\pi$-sets of $D \subset Y$ relative to $T$, and $f=g \mid A \cap B$. We define $h(x)=f(x)$ for $x \in A$ and $h(x)=g(x)$ for $x \in B$. Since $A$ and $B$ are closed, $h$ is clearly continuous on $A \cup B$ and can be extended, by the Tietze extension theorem, to an element of $C(X)$. Then $T f=T h=T g \mid D$, and $A \cap B$ is a $\pi$-set of $D$.

It is easily seen that a necessary and sufficient condition that $T: C(X)$ $\rightarrow C(Y)$ be one-to-one is that $\mu_{T}(Y)=X$. If $X$ is completely regular, and $T$ is one-to-one, then (5) implies that $\pi_{T}(Y)=X$. To see what may happen if $X$ is not completely regular, we take $X$ to be Hewitt's example [2] of a regular space with the property that $C(X)$ consists of the constant functions. Then each point in $X$ is a $\pi$-set of any subset $D$ of $Y$ relative to each $T$ mapping $C(X)$ into $C(Y)$, so that $\pi_{T}(D)=\varnothing$. If $Y=X, D \neq \varnothing$, and $T$ is one-to-one, then $\mu_{T}(D)=X$, and (5) does not hold in this case.

Lemma 2. If $X$ is normal, and $T$ is a nonconstant mapping of $C(X)$ into $C(Y)$ with the property that $Y$ has at least one compact $\pi$-set relative to $T$, then $\pi_{T}(Y) \neq \varnothing$.

Proof. Let $a$ be the collection of $\pi$-sets of $Y$ relative to $T, A_{0} \in Q$ a compact set, and $B=\left\{A \cap A_{0}: A \in Q\right\}$. We have $X \in Q, \varnothing \notin Q$, and $a$ is closed under finite intersections. Hence, $B$ has the finite intersection property, and $\pi_{T}(Y)=\bigcap\{B: B \in B\} \neq \varnothing$.

It may happen that $\pi_{T}(D)=\varnothing$ for $D \neq Y$ even when $X$ is compact. If we choose $A \subset X, f_{0} \in C(X)$ satisfying $f_{0}=\theta \mid A$ and $f_{0} \neq \theta$, and define $T f=f_{0} f$, then $\pi_{T}(A)=\varnothing$.

THEOREM 1. Suppose $X$ is normal, $D \subset Y$, and $T$ is a mapping of $C(X)$ into $C(Y)$ such that, for each $g \in C(X),\{h \in C(X): T g=T h \mid D\}$ is closed in the 
topology $J_{u}$ of uniform convergence on compact subsets for $C(X)$. Then $\pi_{T}(D)$ is a $\pi$-set of $D$ relative to $T$.

Proof. Suppose $f=g \mid \pi_{T}(D), \epsilon>0$, and $K$ is an arbitrary compact subset of $X$. Let $U$ be a neighborhood of $\pi_{T}(D)$ such that $x \in U$ implies $|f(x)-g(x)|$ <є. If $a$ is the collection of $\pi$-sets of $D$ relative to $T$, then, as was shown in Lemma $1, a$ is closed under finite intersections. Since the family $\{(K-U) \cap A: A \in Q\}$ can not have the finite intersection property, there is an $A \in Q$ satisfying $K \cap A \subset U$. We can construct $g_{0} \in C(X)$ so that $g_{0}=\theta\left|A, g_{0}=u\right| K-U$, and $\theta \leqq g_{0} \leqq u$. If we set $f_{0}=(f-g) g_{0}+g$, then $f_{0}=g \mid A$, so that $T f_{0}=T g \mid D$. In addition,

$$
\left|f(x)-f_{0}(x)\right|=|f(x)-g(x)|\left(1-g_{0}(x)\right)\left\{\begin{array}{lr}
<\epsilon & \text { for } x \in U, \\
=0 & \text { for } x \in K-U .
\end{array}\right.
$$

Thus $x \in K$ implies $\left|f(x)-f_{0}(x)\right|<\epsilon$. Since $\epsilon$ and $K$ were arbitrary, $\{h \in C(X): T g=T h \mid D\}$ is closed in $J_{u}$, and $f_{0}$ belongs to this set, we have $T f=T g \mid D$, and $\pi_{T}(D)$ is a $\pi$-set of $D$ relative to $T$.

Corollary. If $X$ is normal, and $T$ is continuous when $C(X)$ is given $\mathfrak{J}_{u}$ and $C(Y)$ a topology $\mathrm{I}$ which is finer than the topology of pointwise convergence, then $\pi_{T}(D)$ is a $\pi$-set of $D$ relative to $T$ for any $D \subset Y$.

Proof. We have only to observe that $\{g \in C(Y): f=g \mid D\}$ is closed in $\mathrm{J}$ for each $f \in C(Y)$.

THEOREM 2. Let $X$ be completely regular, $D \subset Y$, and $T$ a mapping of $C(X)$ into $C(Y)$ with the property that, for any $f, g, h \in C(X), T f=T g \mid D$ implies $T(f+h)=T(g+h) \mid D$ and $T(f h)=T(g h) \mid D$. Then $\mu_{T}(D)=\pi_{T}(D)$.

Proof. Let $A=Z\left(f_{0}-g_{0}\right)$, where $T f_{0}=T g_{0} \mid D$, and let $U$ be a neighborhood of $A$. Then $T\left(f_{0}-g_{0}\right)=T\left(g_{0}-g_{0}\right)=T \theta \mid D$. Suppose $f, g \in C(X)$ satisfy $U \subset Z(f-g)$. If we define $h(x)=(f(x)-g(x)) /\left(f_{0}(x)-g_{0}(x)\right)$ for $x \in X-A$ and $\boldsymbol{h}(x)=0$ for $x \in A$, then $h$ is evidently continuous at each point of $U$ and of $X-A$, so that $h \in C(X)$. Hence, $T \theta=T(\theta h)=T\left(\left(f_{0}-g_{0}\right) h\right)=T(f-g) \mid D$, and $T f=T(f-g+g)=T(\theta+g)=T g \mid D$. Thus $\bar{U}$ is a $\pi$-set of $D$ relative to $T$. Suppose $x \notin \mu_{T}(D)$. We infer from (3) that, for some $f^{\prime}, g^{\prime} \in C(X), T f^{\prime}=T g^{\prime} \mid D$ and $x \notin Z\left(f^{\prime}-g^{\prime}\right)$. Since $X$ is regular, there is a neighborhood $U$ of $Z\left(f^{\prime}-g^{\prime}\right)$ such that $x \notin \bar{U}$. From the preceding discussion, it follows that $x \notin \pi_{T}(D)$. Hence, $\mu_{T}(D) \supset \pi_{T}(D)$, and the theorem follows from (5).

Corollary. If $X$ is normal, $D \subset Y$, and $T$ maps $C(X)$ into $C(Y)$, then the following conditions are equivalent: for any $f, g, h \in C(X)$,

(a) $T f=T g \mid D$ implies $T(f+h)=T(g+h) \mid D$ and $T(f h)=T(g h) \mid D$, and $\{k \in C(X): T f=T k \mid D\}$ is closed in $J_{u}$,

(b) For some closed $A \subset X, T f=T g \mid D$ if, and only if, $f=g \mid A$,

(c) $\mu_{T}(D)=\pi_{T}(D)$, and $\pi_{T}(D)$ is a $\pi$-set of $D$ relative to $T$. 
Proof. Theorems 1 and 2 show that (a) implies (c). That (c) implies (b) and (b) implies the first half of (a) is easily seen. That (b) implies the second half of (a) follows as in the proof of the corollary to Theorem 1.

3. The linear case. If we regard $C(X)$ as a linear space under pointwise addition and scalar multiplication, then most of the preceding results take on a somewhat simpler form when $T$ is assumed to be a linear transformation of $C(X)$ into $C(Y)$. For a linear $T$, the sets $A$ in (1) and (2) can be defined, respectively, as: for all $f \in C(X), T f=\theta \mid D$ implies $f=\theta|A ; f=\theta| A$ implies $T f=\theta \mid D$. The right side of (3) now reads $\cap\{Z(f): T f=\theta \mid D\}$. We can also make the obvious modification in the definition of $\pi$-set. In Theorem 1 , we need only assume that $\{h \in C(X): T h=\theta \mid D\}$ is closed. In Theorem 2, $T(f+h)=T(g+h) \mid D$ is automatically satisfied, and $T(f h)=T(g h) \mid D$ says that $\{f \in C(X): T f=\theta \mid D\}$ is an ideal in $C(X)$.

Lemma 3. For any closed subsets $A$ and $D$ of a completely regular space $X$ with $A \subset D$, there is a linear transformation $T$ of $C(X)$ into itself satisfying $\mu_{T}(D)=\pi_{T}(D)=A$.

Proof. Since $M=\{f \in C(X): f=\theta \mid A\}$ is a linear subspace of $C(X)$, we can find a Hamel basis $\left\{f_{p}: p \in P\right\}$ (over the real field) for $C(X)$ with the property that, for some $Q \subset P,\left\{f_{p}: p \in Q\right\}$ is a Hamel basis for $M$. We now define $T f_{p}=\theta$ for $p \in Q, T f_{p}=f_{p}$ for $p \in P-Q$, and extend $T$ linearly to the rest of $C(X)$. Since $T f_{p}=f_{p} \mid A$ for all $p \in P$, we have $T f=f \mid A$ for all $f \in C(X)$. If $T f=\theta \mid D$, then $T f=\theta \mid A$, and $f=\theta \mid A$, whence $A \subset \mu_{T}(D)$. If $f=\theta \mid A$, then $f \in M, T f=\theta \mid D$, and $A$ is a $\pi$-set of $D$ relative to $T$. The result now follows from (5). We note that if $D=X$, then the null space of $T$ is precisely $M$.

Several relevant examples are obtainable from the following construction. Let $X$ be a topological space, and $a$ a family of closed subsets of $X$ with the property that, for any $A_{1}, A_{2} \in Q$, there is an $A_{3} \in Q$ such that $A_{3} \subset A_{1} \cap A_{2}$. We set $M=\{f \in C(X): Z(f) \supset A$ for some $A \in a\}$. Since $M$ is a linear subspace of $C(X)$, we can find a Hamel basis $\left\{f_{p}: p \in P\right\}$ over the real field for $C(X)$ and a subset $Q$ of $P$ such that $\left\{f_{p}: p \in Q\right\}$ is a Hamel basis for $M$. We now define $T f_{p}=\theta$ for $p \in Q, T f_{p}=f_{p}$ for $p \in P-Q$, and extend $T$ linearly to a mapping of $C(X)$ into itself.

Lемма 4. If $X$ is completely regular, and $T$ is the mapping defined above, then $\mu_{T}(X)=\pi_{T}(X)=\bigcap\{A: A \in Q\}, T^{-1} \theta=M$, and $T^{2}=T$.

Proof. Clearly each $A \in Q$ is a $\pi$-set of $X$ relative to $T$, so that $\pi_{T}(X)$ $\subset \cap\{A: A \in Q\}=A_{0}$. For any $f \in C(X)$, if $f \in M$, then $f=\sum_{p \in F} \alpha_{p} f_{p}$, where $F \subset P$ is finite, and $\alpha_{p} \neq 0$ is real. Moreover, $F \cap(P-Q) \neq \varnothing$, so that $T f \neq \theta$. Since $T(M)=\{\theta\}$, we have $T^{-1} \theta=M$. We infer from (3) that $A_{0} \subset \mu_{T}(X)$, and from (5) that $\mu_{T}(X)=\pi_{T}(X)=A_{0}$. Since $T^{2} f_{p}=T f_{p}$ for all $p \in P$, we have $T^{2}=T$.

Example 1. We will exhibit a linear mapping $T$ of $C(X)$ into itself with 
the property that $\pi_{T}(X)$ is not a $\pi$-set of $X$ relative to $T$. Suppose $X$ is completely regular, and there is a $g \in C(X)$ such that $\varnothing \neq Z(g) \neq X$, and $Z(g)$ is not open. Let $a$ be the family of closed neighborhoods of $Z(g)$, and $T$ the mapping defined above. From the complete regularity of $X$ and Lemma 4, we have $\pi_{T}(X)=\bigcap\{A: A \in \mathrm{Q}\}=Z(\mathrm{~g})$, but $g \notin M$, so that $\pi_{T}(X)$ is not a $\pi$-set of $X$ relative to $T$.

4. Finite dimensional ranges. We now turn to the properties of $\mu_{T}$ and $\pi_{T}$ when $T$ is linear and its range $R(T)$ is finite-dimensional. It is, of course, true of any linear transformation $T$ of $C(X)$ into $C(Y)$ (or of one linear space into another) that $R(T)$ is finite-dimensional if, and only if, $T f=\sum_{i=1}^{n} L_{i}(f) g_{i}$ for all $f \in C(X)$, where $L_{i}$ is a linear functional on $C(X)$, and the $g_{i} \in C(Y)$ are linearly independent.

TheOREM 3. If $X$ is normal, and $T$ is a linear transformation of $C(X)$ into $C(Y)$, then the following are equivalent:

(a) $\pi_{T}(Y)$ is finite and a $\pi$-set of $Y$ relative to $T$,

(b) $\pi_{T}(Y)$ is finite, and $T^{-1} \theta$ is closed in $J_{u}$,

(c) $T f=\sum_{i=1}^{n} f\left(x_{i}\right) g_{i}$, where the $x_{i} \in X$ and $g_{i} \in C(Y)$ are fixed.

These conditions imply that $R(T)$ is finite-dimensional.

Proof. We first show that (a) implies (c). If $\pi_{T}(Y)=\varnothing$, then $T(C(X))$ $=\{\theta\}$, and (c) holds with the $g_{i}=\theta$. Suppose $\pi_{T}(Y)=\left\{x_{1}, \cdots, x_{n}\right\}$. For each $i, j(1 \leqq i, j \leqq n)$, choose $f_{i} \in C(X)$ such that $f_{i}\left(x_{j}\right)=\delta_{i j}$ (the Kronecker $\delta$ ), and set $g_{i}=T f_{i}$. If $f \in C(X)$, then $\sum_{i=1}^{n} f\left(x_{i}\right) f_{i}=f \mid \pi_{T}(Y)$, and $T f=\sum f\left(x_{i}\right) g_{i}$. We next show that (c) implies (b). Clearly $\left\{x_{1}, \cdots, x_{n}\right\}$ is a $\pi$-set of $Y$ relative to $T$. The mapping $\alpha \rightarrow \alpha g_{i}$ from $E_{1}$ into $C(Y)$ is evidently continuous in $\mathfrak{J}_{u}$. Since addition in $C(Y)$ is continuous in $J_{u}$, the mapping $\left(\alpha_{1}, \cdots, \alpha_{n}\right)$ $\rightarrow \sum_{i=1}^{n} \alpha_{i} g_{i}$ from $E_{n}$ into $C(Y)$ is also continuous. Finally, the mapping $f \rightarrow f\left(x_{i}\right)$ from $C(X)$ with topology $J_{u}$ into $E_{1}$ is continuous. Since $T f=\sum f\left(x_{i}\right) g_{i}$, $T$ is continuous, and $T^{-1} \theta$ is closed in $J_{u}$. That (b) implies (a) follows from Theorem 1. The last statement of the theorem is obvious.

Corollary. If $X$ is normal, and $T$ satisfies (c) above, then the dimension of $\mathbb{R}(T)$ does not exceed the cardinality of $\pi_{T}(Y)$.

Proof. Suppose $x_{j} \notin \pi_{T}(Y)$ for some $j, 1 \leqq j \leqq n$. Then there is a $\pi$-set $A$ of $Y$ relative to $T$ such that $x_{j} \notin A$. Since $\left\{x_{1}, \cdots, x_{n}\right\}$ is a $\pi$-set of $Y$, $A \cap\left\{x_{1}, \cdots, x_{n}\right\}=B$ is also one. From $f_{j}=\theta \mid B$ we infer that $\theta=T f_{j}=g_{j}$, and the inequality follows immediately.

LEMMA 5. If $X$ is completely regular, and $T$ is a linear transformation of $C(X)$ into $C(Y)$ whose range has dimension $n<\infty$, then $\mu_{T}(Y)$ has at most $n$ points.

Proof. Suppose that $\mu_{T}(Y) \supset\left\{x_{1}, \cdots, x_{n+1}\right\}$. Choose $f_{1}, \cdots, f_{n+1} \in C(X)$ satisfying $f_{i}\left(x_{j}\right)=\delta_{i j}(1 \leqq i, j \leqq n+1)$, and set $g_{i}=T f_{i}$. Then there will be real 
numbers $\alpha_{i}$, not all 0 , such that $\sum_{i=1}^{n+1} \alpha_{i} g_{i}=\theta$. Setting $f=\sum_{i=1}^{n+1} \alpha_{i} f_{i}$, we have $T f=\theta$, whence $f=\theta \mid \mu_{T}(Y)$. But $f\left(x_{i}\right)=\alpha_{i}$, a contradiction. Hence, $\mu_{T}(Y)$ has at most $n$ points.

EXAMPLE 2. We will exhibit a mapping $T$ of $C(X)$ into itself such that $R(T)$ is finite dimensional, and $\pi_{T}(X)$ is infinite. Suppose $X$ is a completely regular space with a base $\left\{U_{p}: p \in R\right\}$ having the following properties:

(i) For every finite $F \subset R$, there is a $q \in F$ such that $U_{q}$ $\nsubseteq \cup\left\{U_{p}: p \in F-\{q\}\right\}$,

(ii) For each $p \in R$, there is an $f_{p} \in C(X)$ satisfying $Z\left(f_{p}\right)=X-U_{p}$. We may, for example, take $X=E_{n}$ and $\left\{U_{p}\right\}$ to be the set of open $n$-balls. To see that (i) is satisfied, let II be a hyperplane of support for $U\left\{U_{p}: p \in F\right\}$. Then for some $p \in F, \Pi$ is tangent to $\bar{U}_{p}$ at a point $x$. Let $U_{q}, q \in F$, be the ball of largest radius which is tangent to $\Pi$ at $x$. There is clearly no difficulty in satisfying (ii) in this case. Returning to the general case, $\left\{f_{p}: p \in R\right\}$ is linearly independent. For if $F \subset R$ is finite, and $\sum_{p \in F} \alpha_{n} f_{p}=\theta$ for real $\alpha_{p} \neq 0$, then

$$
\theta=\sum_{p \in F} \alpha_{p} f_{p}=\alpha_{q} f_{q} \mid U_{q}-U\left\{U_{p}: p \in F-\{q\}\right\}
$$

and $\alpha_{q}=0$, which is a contradiction.

Let $A \neq \varnothing$ be a subset of $X$ which is the closure of its interior, $M=\{f \in C(X): f=\theta \mid A\}$, and $R_{0}=\left\{p \in R: U_{p} \subset A\right\}$. The above reasoning shows that the linear subspace spanned by $\left\{f_{p}: p \in R_{0}\right\}$ intersects $M$ in $\{\theta\}$. Hence, we can find a Hamel basis $\left\{f_{p}: p \in P\right\}$ for $C(X)$ such that $R_{0} \subset P$ and, for some $Q \subset P-R_{0},\left\{f_{p}: p \in Q\right\}$ is a Hamel basis for $M$. We choose $f_{0}$ from $\left\{f_{p}: p \in R_{0}\right\}$ and define $T f_{p}=f_{0}$ for $p \in P-Q, T f_{p}=\theta$ for $p \in Q$, extending $T$ linearly to $C(X)$. If $D \supset A$ is a fixed closed subset of $X$, then $A$ is evidently a $\pi$-set of $D$ relative to $T$. If $B$ is a $\pi$-set of $D$ relative to $T$, and $A-B$ $\neq \varnothing$, then we can find $p \in R_{0}$ satisfying $U_{p} \subset A-B$, so that $f_{p}=\theta \mid B$ and $T f_{p}$ $=\theta \mid D$. But $T f_{p}=f_{0}$, which is a contradiction. Hence, $B \supset A$, and $\pi_{T}(D)=A$. We will also show that $\mu_{T}(D)=\varnothing$. Suppose $x \in \mu_{T}(D)$. Then for each $p \in P-Q$, we have $T f_{p}=T f_{0}$, whence $f_{p}(x)=f_{0}(x)$. We can evidently find $q \in R_{0}$ such that $x \in X-U_{q}$, so that $f_{0}(x)=f_{q}(x)=0$. From (5), $x \in A$, and $f_{p}(x)=0$ for all $p \in P$. But this is impossible, since $\left\{f_{p}: p \in P\right\}$ spans $C(X)$. Therefore, $\mu_{T}(D)=\varnothing$.

The range of $T$ is, of course, one-dimensional. Thus we see that $\pi_{T}(X)$ can be infinite while $R(T)$ is finite dimensional, the dimension can be greater than the cardinality of $\mu_{T}(X)$, and the converse of Theorem 3 is not valid.

EXAmple 3. The above construction can be extended to give an example of a mapping $S$ of $C(X)$ onto itself such that $\pi_{S}(\{x\})=X$ and $\mu_{S}(\{x\})=\varnothing$ for each $x \in X$. Let $X=E_{n},\left\{x_{p}: p \in J\right\}$ be a dense subset of $E_{n}$, where $J$ is the set of positive integers, and, for each $p, q \in J, U_{p q}$ the open ball with center $x_{p}$ and radius $1 / q$. Clearly $\left\{U_{p q}: p, q \in J\right\}$ satisfies (i), and we can easily find $f_{p q} \in C(X)$ satisfying $Z\left(f_{p q}\right)=X-U_{p q}$, so that (ii) is also realized. We now 
define $S f_{p q}=f_{q p}$ for each $p, q \in J$. Since $\left\{f_{p q}: p, q \in J\right\}$ is linearly independent, it can be extended to a Hamel basis for $C(X)$. We make $S$ leave each of these additional basis elements fixed and extend $S$ to a linear mapping of $C(X)$ onto itself which is, in fact, an involution. Let $x$ be a point of $X$ and $A \neq X$ a closed subset of $X$. Choose $y \in X-A$, an open ball $U$ with center at $y$ and radius $\epsilon$ satisfying $U \subset X-A$, and $k \in J$ such that $2 / k<\epsilon$. Then we can find an $m \geqq k$ for which $\left|x_{m}-y\right|<1 / k$. Since $\left\{U_{q m}: q \geqq m\right\}$ is a covering of $X$, there is an $r \geqq m$ satisfying $x \in U_{r m}$. From $r \geqq k$ we infer that $U_{m r} \subset U \subset X$ $-A$, and $A \subset Z\left(f_{m r}\right)$. But $S f_{m r}(x)=f_{r m}(x) \neq 0$, so that $A$ is not a $\pi$-set of $\{x\}$ relative to $S$. Hence, $\pi_{S}(\{x\})=X$. If we set $A=\{z\}$, for some $z \in X$, in the above construction, then $S f_{r m}(z)=f_{m r}(z)=0$, and $f_{r m}(x) \neq 0$. Thus $x \notin \mu_{S}(\{z\})$, and $\mu_{S}(\{z\})=\varnothing$.

EXAMPLE 4. It was observed in $\$ 2$ that if $X$ is completely regular, and $T: C(X) \rightarrow C(Y)$ is one-to-one, then $\pi_{T}(Y)=X$. To show that the converse does not hold in general, we set $A=D=X$ in Example 2. Then $\pi_{T}(X)=X$, $Q=\varnothing$, and $T f_{p}=f_{0}$ for all $p \in P$, so that $T$ is not one-to-one.

EXAMPLE 5. We will show that there are mappings $T$ of $C(X)$ into itself for which $\pi_{T}(X)=\varnothing$ and $R(T)$ is not finite dimensional. Let $X=E_{1}, a$ be the family of closed subsets of $X$ with relatively compact complements, and $T$ be defined as in the construction preceding Lemma 4. From Lemma 4, $\pi_{T}(X)=\bigcap\{A: A \in Q\}=\varnothing$. We will show that $P-Q$ is infinite. Suppose $\left\{f_{p}: p \in P-Q\right\}=\left\{f_{1}, \cdots, f_{n}\right\}$, and set $g_{i}(x)=x^{i}$ for $1 \leqq i \leqq n$. For any $f \in C(X)$, we have $f=\sum\left\{\alpha_{p} f_{p}: p \in F\right\}$, where $F \subset P$ is finite, and $\alpha_{p}$ is real. For each $p \in Q, Z\left(f_{p}\right)$ contains some $A_{p} \in Q$. If we set $B=\cap\left\{A_{p}: p \in F \cap Q\right\}$, then $B \in Q$, and $f=\sum\left\{\alpha_{p} f_{p}: p \in F \cap(P-Q)\right\} \mid B$. Consequently, we can find $A_{i} \in Q$ and real $\alpha_{i j}$, not all 0 , such that $g_{i}=\sum_{j=1}^{n} \alpha_{i j} f_{j} \mid A_{\imath}$ for $1 \leqq i \leqq n$. Since the $g_{i} \mid A$ are linearly independent for any $A \in Q$, there are real $\beta_{i j}$ such that $f_{j}=\sum_{i=1}^{n} \beta_{i j} g_{i} \mid A_{0}$, where $A_{0}=\bigcap_{i=1}^{n} A_{i}$, and $1 \leqq j \leqq n$. Let $g_{0} \neq \theta$ be a bounded element of $C(X)$. Since $g_{0}=\sum \gamma_{j} f_{j} \mid B_{1}$ for some $B_{1} \in Q$, we have $g_{0}=\sum \delta_{i} g_{i} \mid B_{2}$ for some $B_{2} \in Q$, which is impossible. Hence, $P-Q$ is infinite, and $R(T)$ is not finite dimensional. We see from this example that the condition in Theorem 3 (a) that $\pi_{T}(Y)$ is a $\pi$-set of $Y$ relative to $T$ cannot be dropped.

5. Comparison of maximum values. To avoid treating special cases when taking the supremum of the empty set in the following sections, we introduce the following notation. If $A$ and $B$ are sets of non-negative real numbers, we define

$$
\operatorname{Sup}\{x: x \in A\}= \begin{cases}\sup \{x: x \in A\} & \text { if } A \neq \varnothing, \\ 0 & \text { if } A=\varnothing,\end{cases}
$$

where sup denotes the ordinary operation. The following relations are easily checked:

$$
\begin{aligned}
A \subset B \text { implies } \operatorname{Sup}\{x: x \in A\} & \leqq \operatorname{Sup}\{x: x \in B\}, \\
\operatorname{Sup}\{\beta x: x \in A\} & =\beta \operatorname{Sup}\{x: x \in A\}, \quad \beta \geqq 0,
\end{aligned}
$$


$\operatorname{Sup}\{x+y: x \in A, y \in B\} \leqq \operatorname{Sup}\{x: x \in A\}+\operatorname{Sup}\{y: y \in B\}$, $\operatorname{Sup}\{x y: x \in A, y \in B\} \leqq \operatorname{Sup}\{x: x \in A\} \operatorname{Sup}\{y: y \in B\}$.

Let $X$ and $Y$ be topological spaces, $T$ an arbitrary mapping of $C(X)$ into $C(Y), K$ a compact subset of $Y$, and $\beta$ a real number $\geqq 0$. In this section we will assume that $Y$ is a Hausdorff space. We define $a$ to be the family of closed subsets of $A X$ with the property that, for all $f, g \in C(X)$,

(6) $\beta \operatorname{Sup}\{|f(x)-g(x)|: x \in A\} \geqq \operatorname{Sup}\{|T f(y)-T g(y)|: y \in K\}$.

If $a$ is not empty, we set

$$
\underset{\rho}{\beta}(K)=\cap\{A: A \in \mathrm{Q}\} ;
$$

otherwise, we leave $\rho_{T}^{\beta}(K)$ undefined. The members of $a$ will be called $\rho$-sets of $K$ relative to $T$ and $\beta$. Since any closed set containing a $\rho$-set of $K$ is also a $\rho$-set of $K$, it follows that $\rho_{T}^{\beta}(K)$ is defined if, and only if, $X$ is a $\rho$-set of $K$. Since $\operatorname{Sup}\{|f(x)|: x \in \varnothing\}=0, \varnothing$ is a $\rho$-set of $\varnothing$ relative to any $T$ and $\beta$, and $\rho_{T}^{\beta}(\varnothing)=\varnothing$. If $T$ is a constant mapping, then $\varnothing$ is a $\rho$-set of $K$ relative to $T$ and any $\beta$, and $\rho_{T}^{\beta}(K)=\varnothing$.

Lemma 6. If $T$ maps $C(X)$ into $C(Y)$, and $\rho_{T}^{\beta}(K)$ is defined for some compact $K \subset Y$ and $\beta \geqq 0$, then the $\pi$-sets of $K$ relative to $T$ coincide with the $\rho$-sets of $K$ relative to $T$ and $\beta$, and $\rho_{T}^{\beta}(K)=\pi_{T}(K)$.

Proof. Clearly every $\rho$-set of $K$ relative to $T$ and $\beta$ is a $\pi$-set of $K$ relative to $T$. Let $B$ be a $\pi$-set of $K$. For any $f, g \in C(X)$, we set $m=\operatorname{Sup}\{|f(x)-g(x)|$ : $x \in B\}$ and $f_{1}=(g-m u) \vee((g+m u) \wedge f)$; if $m=\infty$, we let $f_{1}=f$. Then $f_{1}=f \mid B$, $T f_{1}=T f|K,| f_{1}-g \mid \leqq m u$, and

$$
\begin{aligned}
\beta \operatorname{Sup} & \{|f(x)-g(x)|: x \in B\} \geqq \beta \operatorname{Sup}\left\{\left|f_{1}(x)-g(x)\right|: x \in X\right\} \\
& \geqq \operatorname{Sup}\left\{\left|T f_{1}(y)-T g(y)\right|: y \in K\right\}=\operatorname{Sup}\{|T f(y)-T g(y)|: y \in K\} .
\end{aligned}
$$

Hence, $B$ is a $\rho$-set of $K$, and the families of $\pi$-sets and $\rho$-sets coincide.

Lemma 7. If $X$ is normal, $K$ a compact subset of $Y$, and $T$ is a mapping of $C(X)$ into $C(Y)$ with the property that, for some $\beta \geqq 0, K$ has at least one compact $\rho$-set relative to $T$ and $\beta$, then $\pi_{T}(K)$ is a $\pi$-set of $K$ relative to $T$.

Proof. Let $a$ be the family of $\rho$-sets of $K$ relative to $T$ and $\beta$, and $A_{0} \in Q$ a compact set. If $A_{0}=\varnothing$, then $\pi_{T}(K)=\varnothing$, and the conclusion is certainly valid, so we will assume that $A_{0} \neq \varnothing$. Since $A_{0}$ is a $\pi$-set of $K$ relative to $T$, the mapping $T^{\prime}$ from $\left\{f \mid A_{0}: f \in C(X)\right\}$ into $\{g \mid K: g \in C(Y)\}$ defined by $T^{\prime}\left(f \mid A_{0}\right)=T f \mid K$ is single-valued. From the compactness of $A_{0}$ we infer that $\left\{f \mid A_{0}: f \in C(X)\right\}=C\left(A_{0}\right)$; thus $T^{\prime}$ maps $C\left(A_{0}\right)$ into $C(K)$. Since $\pi_{T}(K)$ is the intersection of a family of sets which includes $A_{0}$, we have $\pi_{T^{\prime}}(K)=\pi_{T}(K)$. Now if $\pi_{T}(K)$ is empty, we conclude from Lemma 2, with $X=A_{0}$ and $Y=K$, that $T^{\prime}$ is constant. Hence, $\varnothing$ is a $\pi$-set of $K$ relative to $T^{\prime}$, and thus also 
relative to $T$, and Lemma 7 is verified in this case. We now assume $\pi_{T}(K) \neq \varnothing$. Let $B=\left\{A \cap A_{0}: A \in Q\right\}$. Since the $\pi$-sets and $\rho$-sets of $K$ coincide, $B$ is closed under finite intersections. Suppose $f, g \in C(X)$ satisfy

$$
\begin{aligned}
\gamma & =\beta \operatorname{Sup}\left\{|f(x)-g(x)|: x \in \pi_{T}(K)\right\} \\
& <\operatorname{Sup}\{|T f(y)-T g(y)|: y \in K\}=\delta .
\end{aligned}
$$

Then we can find a neighborhood $U$ of $\pi_{T}(K)$ such that $x \in U$ implies $\beta|f(x)-g(x)|<(\gamma+\delta) / 2$. Since $\pi_{T}(K)=\bigcap\{B: B \in \mathbb{B}\}$, and the members of $\mathbb{B}$ are compact, there is a $B_{0} \in ß$ satisfying $B_{0} \subset U$. But then

$$
\beta \operatorname{Sup}\left\{|f(x)-g(x)|: x \in B_{0}\right\}<\delta,
$$

which is impossible. We conclude, therefore, that $\pi_{T}(K)$ is a $\rho$-set of $K$ relative to $T$ and $\beta$ and, hence, a $\pi$-set of $K$ relative to $T$.

We turn now to the case where $T$ is a linear transformation of $C(X)$ into $C(Y)$ and observe that (6) can now be written as

$$
\beta \operatorname{Sup}\{|f(x)|: x \in A\} \geqq \operatorname{Sup}\{|T f(y)|: y \in K\} .
$$

Example 6. We will exhibit a $T$ mapping $C(X)$ into itself with the property that $\rho_{T}^{\boldsymbol{\beta}}(K)$ is not defined for any compact $K \neq \varnothing$ and any $\beta \geqq 0$. Let $X=E_{n}, R$ be the set of positive integers, and $\left\{U_{p}: p \in R\right\}$ the family of open $n$-balls with radius $r_{p}$ a positive rational number and center $x_{p}$ having rational coordinates. For each $p \in R$, we define

$$
f_{p}(x)= \begin{cases}1-\left|x-x_{p}\right| / r_{p} & \text { for }\left|x-x_{p}\right| \leqq r_{p}, \\ 0 & \text { for }\left|x-x_{p}\right| \geqq r_{p} .\end{cases}
$$

Using the reasoning in Example 4, we see that $\left\{U_{p}: p \in R\right\}$ satisfies (i) and (ii), and $\left\{f_{p}: p \in R\right\}$ is linearly independent. Hence, the latter can be extended to a Hamel basis $\left\{f_{p}: p \in P\right\}$ for $C(X)$, where $R \subset P$. We now define $T f_{p}=p f_{p}$ for $p \in R$, and $T f_{p}=f_{p}$ for $p \in P-R$, extending $T$ linearly to $C(X)$. To show that $\rho_{T}^{\beta}(K)$ is not defined for any compact $K \neq \varnothing$ and any $\beta \geqq 0$ is evidently equivalent to showing that, for each $x \in X, X$ is not a $\rho$-set of $\{x\}$ relative to $T$ and any $\beta \geqq 0$. If $x$ and $\beta$ are given, we can find $p, q \in R$ satisfying $1+\beta \leqq p$ $\leqq q, r_{q}=1$, and $\left|x_{q}-x\right|<1 / p$. Then

$$
\left|T f_{q}(x)\right|=q f_{q}(x)>q(1-1 / p) \geqq p(1-1 / p) \geqq \beta=\beta \operatorname{Sup}\left\{\left|f_{q}(y)\right|: y \in X\right\} \text {, }
$$

and our assertion is proved. Therefore, $\rho_{T}^{\beta}(K)$ is undefined unless $K=\varnothing$.

6. Power series. Suppose $X$ is a topological space, and $T_{1}, \cdots, T_{k}$ map $C(X)$ into itself. We will set $\pi_{T_{i}}=\pi_{i}$ and $\rho_{T_{i}}^{\beta}=\rho_{i}^{\beta}$ for $i=1, \cdots, k$. By taking all possible compositions of the $\pi_{i}$ with any finite number of factors, we obtain a semigroup $\Psi\left(\pi_{1}, \cdots, \pi_{k}\right)$ of mappings of $2^{X}$ into itself. For any $D \subset X$, we define 


$$
\sigma(D)=\left(\bigcup\left\{\psi(D): \psi \in \Psi\left(\pi_{1}, \cdots, \pi_{k}\right)\right\}\right)^{-},
$$

where the bar at the right denotes closure.

For each integer $m \geqq 1$, let $P_{m}\left(t_{1}, \cdots, t_{k}\right)$ be a homogeneous polynomial of degree $m$ with real coefficients in the indeterminates $t_{1}, \cdots, t_{k}$, where $k \geqq 1$ is fixed, the $t_{i}$ are not assumed to commute, and terms with the same factors in different orders are counted as distinct. We denote by $c_{m}$ the sum of the absolute values of the coefficients of $P_{m}$.

We recall that a $k$-space $[3$, p. 230] is a topological space with the property that if any subset $A$ intersects each closed, compact subset in a closed set, then $A$ is closed. Among the $k$-spaces are all Hausdorff spaces which are either first countable or locally compact.

TheOREM 4. Let $X$ be a Hausdorff $k$-space, and $T_{1}, \cdots, T_{k}$ map $C(X)$ into itself in such a way that $T_{i} \theta=\theta, i=1, \cdots, k$. Suppose that, for each compact $K \subset X$ and each $i, 1 \leqq i \leqq k, \pi_{i}(K)$ is a $\pi$-set of $K$ relative to $T_{i}$, there is a real $\beta$, $0 \leqq \beta<1$, such that $\rho_{i}^{\beta}(K)$ is defined, and $\sigma(K)$ is compact. Finally, suppose $P_{m}$ as defined above has the property that $\sum_{m=1}^{\infty} c_{m} t^{m}$ converges for $|t|<1$. Then $\sum_{m=1}^{\infty} P_{m}\left(T_{1}, \cdots, T_{k}\right) f$ converges in the topology $J_{u}$ to an element of $C(X)$ for each $f \in C(X)$.

Proof. Let $K \subset X$ be compact. Since $\sigma(K)$ is compact, each $\rho_{i}^{\beta}(K)$ is defined for some $\beta=\beta_{i}$, and each $\rho_{i}^{\beta}(\sigma(K))$ for some $\beta=\gamma_{i}$. If we set $\beta_{0}=\sup \left\{\beta_{1}, \cdots, \beta_{k}, \gamma_{1}, \cdots, \gamma_{k}\right\}$, then $\rho_{i}^{\beta}(K)$ and $\rho_{i}^{\beta}(\sigma(K))$ are defined for $\beta=\beta_{0}<1$. We will show by induction on $m$ that if $T_{p} \cdots T_{q}$ consists of $m$ factors from among $T_{1}, \cdots, T_{k}$, then

(8) $\operatorname{Sup}\left\{\left|T_{p} \cdots T_{q} f(y)\right|: y \in K\right\} \leqq \beta_{0}^{m} \operatorname{Sup}\{|f(x)|: x \in \sigma(K)\}, f \in C(X)$.

If $m=1$, we infer from Lemma 6 that $\pi_{p}(K)$ is a $\rho$-set of $K$ relative to $T_{p}$ and $\beta_{0}$, and since $T_{p} \theta=\theta$, (7) holds with $T=T_{p}, \beta=\beta_{0}$, and $A=\pi_{p}(K)$. Then $\pi_{p}(K) \subset \sigma(K)$ implies

$$
\operatorname{Sup}\left\{|f(x)|: x \in \pi_{p}(K)\right\} \leqq \operatorname{Sup}\{|f(x)|: x \in \sigma(K)\},
$$

and (8) is verified in this case. Suppose (8) is valid for some $m$, and $T_{p} \cdots T_{q} T_{r}$ consists of $m+1$ factors. Then

$$
\begin{aligned}
\operatorname{Sup}\left\{\mid T_{p}\right. & \left.\cdots T_{q} T_{r} f(y) \mid: y \in K\right\} \leqq \beta_{0}^{m} \operatorname{Sup}\left\{\left|T_{r} f(y)\right|: y \in \sigma(K)\right\} \\
& \leqq \beta_{0}^{m+1} \operatorname{Sup}\left\{|f(x)|: x \in \pi_{r}(\sigma(K))\right\} \leqq \beta_{0}^{m+1} \operatorname{Sup}\{|f(x)|: x \in \sigma(K)\},
\end{aligned}
$$

since $\pi_{r}(\sigma(K)) \subset \sigma(K)$. Hence, (8) holds for all $m \geqq 1$. If we set $S_{n}$ $=\sum_{m=1}^{m} P_{m}\left(T_{1}, \cdots, T_{k}\right)$, then for any $x \in K$ and any integers $q \geqq p \geqq 1$,

$$
\left|S_{q} f(x)-S_{p} f(x)\right| \leqq \sum_{m=p+1}^{q} c_{m} \beta_{0}^{m} \operatorname{Sup}\{|f(z)|: z \in \sigma(K)\}, \quad f \in C(X) .
$$


Hence, $\left\{S_{n} f(x): n \geqq 1\right\}$ is a Cauchy sequence with limit $g(x)$, and $\left\{S_{n} f\right\}$ converges to $g$ uniformly on $K$. It follows (cf. [3, p. 231]) that $g \in C(X)$.

EXAMPLE 7. The next theorem concerns a class of mappings $T$ of $C(X)$ into itself of which examples are easily constructed when $X=E_{n}$. Let $\Sigma$ be an $(n-1)$-sphere in $E_{n}$ with center $x_{0}$ and radius $r$, and $\phi$ a continuous, real valued function defined on $\{t: t \geqq 0\}$ with the property that $\phi(0)=1$. For any $f \in C\left(E_{n}\right)$, we define

$$
T f(x)= \begin{cases}f(x) & \text { for }\left|x-x_{0}\right| \leqq r \\ f\left(x_{1}\right) \cdot \phi\left(\left|x-x_{1}\right|\right) & \text { for }\left|x-x_{0}\right| \geqq r\end{cases}
$$

where $x_{1}$ is the intersection of the radial line through $x_{0}$ and $x$ with $\Sigma$. Clearly $T f \in C\left(E_{n}\right)$, znd $T$ is linear. If we let $A$ be the closed ball bounded by $\Sigma$, then $T f=f \mid A$. Now $A$ is a $\pi$-set of $E_{n}$ relative to $T$, and $T^{2}=T$. Moreover, $A \subset \mu_{T}\left(E_{n}\right)$, and from (5), $\mu_{T}\left(E_{n}\right)=\pi_{T}\left(E_{n}\right)=A$. Since $\phi\left(\left|x-x_{1}\right|\right)=T u(x)$ for $\left|x-x_{0}\right| \geqq r$, it follows that if $D$ is a closed subset of $E_{n}$, and $D \subset Z(T u)$, then $\varnothing$ is a $\pi$-set of $D$ relative to $T$. In addition, if $D \subset E_{n}-(A \cup Z(T u))$, then $\pi_{T}(D)$ is simply the radial projection of $D$ on $\Sigma$. For an arbitrary closed subset $D$ of $E_{n}, \pi_{T}(D)$ consists of $D \cap A$ together with the radial projection of $(D-(A \cup Z(T u)))^{-}$on $\Sigma$. Let $K$ be a compact subset of $E_{n}-A$ and

$$
\gamma=\operatorname{Sup}\{|T u(x)|: x \in K\} \text {. }
$$

If $K_{1}$ is the radial projection of $K$ on $\Sigma$, and $f \in C\left(E_{n}\right)$, we have

$$
\begin{aligned}
\operatorname{Sup}\{|T f(x)|: x \in K\} & =\operatorname{Sup}\left\{\left|f\left(x_{1}\right)\right| \cdot\left|\phi\left(\left|x-x_{1}\right|\right)\right|: x \in K\right\} \\
& \leqq \gamma \operatorname{Sup}\left\{|f(y)|: y \in K_{1}\right\} .
\end{aligned}
$$

Hence, $K_{1}$ is a $\rho$-set of $K$ relative to $T$ and $\gamma$, and $\rho_{T}^{\gamma}(K)$ is defined. Thus if $|\phi(t)| \leqq 1$ for $t \geqq 0$, and $K \subset E_{n}$ is compact, then $A$ is a $\rho$-set of $K$ relative to $T$ and 1 , and $\rho_{T}^{1}(K)$ is defined.

For each integer $m \geqq 1$, we define

$$
Q_{m}\left(t_{1}, \cdots, t_{k}\right)=\sum t_{i_{1}} t_{i_{2}} \cdots t_{i_{m}},
$$

where $k \geqq 2$ is fixed, and the sum is extended over all $m$-tuples $\left(i_{1}, \cdots, i_{m}\right)$ chosen from $1, \cdots, k$ with the property that $i_{j} \neq i_{j+1}$ for $j=1, \cdots, m-1$.

TheOREM 5. Let $X$ be a Hausdorff $k$-space, and $T_{1}, \cdots, T_{k}$ map $C(X)$ into itself in such a way that $T_{i} \theta=\theta$ for $i=1, \cdots, k$. Suppose that, for each compact $K \subset X$ and each $i, 1 \leqq i \leqq k, \pi_{i}(K)$ is a $\pi$-set of $K$ relative to $T_{i}, \rho_{i}^{\beta}(K)$ is defined for some $\beta \geqq 0, \sigma(K)$ is compact, and $K \subset \pi_{i}(X)$ implies that $\rho_{j}^{\beta}(K)$ is defined for some $\beta<1 /(k-1)$ whenever $j \neq i$. Finally, suppose there is a closed subset $A_{i}$ of $\pi_{i}(X)$ with the property that $f \in C(X)$ implies $T_{i} f=f \mid A_{i}$ for $i=1, \cdots, k$. Then $\sum_{m=1}^{\infty}(-1)^{m-1} Q_{m}\left(T_{1}, \cdots, T_{k}\right) f=T f$ converges in the topology $J_{u}$ to an element of $C(X)$ for each $f \in C(X)$, and $T f=f \mid \cup_{i=1}^{k} A_{i}$. 
Proof. Let $K \subset X$ be compact. Then $\pi_{i}(K) \subset \pi_{i}(X)$, and $\sigma(K) \subset \bigcup_{i=1}^{k} \pi_{i}(X)$. For each $i \neq j, \rho_{j}^{\beta}\left(\sigma(K) \cap \pi_{i}(X)\right)$ is defined for some $\beta=\alpha_{i j} /(k-1)$, where $0 \leqq \alpha_{i j}<1$. We set $\alpha=\sup \left\{\alpha_{i j}: i, j=1, \cdots, k, i \neq j\right\}$. Since $\beta_{i}^{\beta}(K)$ is defined for some $\beta=\beta_{i}$, it follows that $\rho_{i}^{\beta}(K)$ is defined for $\beta=\sup \left\{\beta_{i}: 1 \leqq i \leqq k\right\}=\beta_{0}$. We will show by induction on $m$ that if $T_{p} \cdots T_{q}$ is a term in $Q_{m}\left(T_{1}, \cdots, T_{k}\right)$ as defined in (9), and $m \geqq 1$, then

$$
\begin{aligned}
\operatorname{Sup}\left\{\left|T_{p} \cdots T_{q} f(y)\right|: y \in K\right\} & \\
& \leqq \frac{\beta_{0} \alpha^{m-1}}{(k-1)^{m-1}} \operatorname{Sup}\left\{|f(x)|: x \in \pi_{q}(X) \cap \sigma(K)\right\}, \quad f \in C(X) .
\end{aligned}
$$

The case $m=1$ follows by definition. Suppose (10) is valid for some $m \geqq 1$, and $T_{p} \cdots T_{q} T_{r}$ is a term in $Q_{m+1}\left(T_{1}, \cdots, T_{k}\right)$. Then $q \neq r, \pi_{r}\left(\pi_{q}(X) \cap \sigma(K)\right)$ $C \pi_{r}(X) \cap \sigma(K), \pi_{q}(X) \cap \sigma(K)$ is compact, and $\pi_{r}\left(\pi_{q}(X) \cap \sigma(K)\right)$ is a $\rho$-set of $\pi_{q}(X) \cap \sigma(K)$ relative to $T_{r}$ and $\alpha /(k-1)$. Hence,

$$
\begin{aligned}
\operatorname{Sup} & \left\{\left|T_{p} \cdots T_{q} T_{r} f(y)\right|: y \in K\right\} \\
& \leqq \frac{\beta_{0} \alpha^{m-1}}{(k-1)^{m-1}} \operatorname{Sup}\left\{\left|T_{r} f(y)\right|: y \in \pi_{q}(X) \cap \sigma(K)\right\} \\
& \leqq \frac{\beta_{0} \alpha^{m}}{(k-1)^{m}} \operatorname{Sup}\left\{|f(x)|: x \in \pi_{r}(X) \cap \sigma(K)\right\},
\end{aligned}
$$

and (10) is established for all $m \geqq 1$. We infer immediately from (10) that

(11) $\operatorname{Sup}\left\{\left|T_{p} \cdots T_{q} f(y)\right|: y \in K\right\} \leqq \frac{\beta_{0} \alpha^{m-1}}{(k-1)^{m-1}} \operatorname{Sup}\{|f(x)|: x \in \sigma(K)\}$.

Since there are $k(k-1)^{m-1}$ terms in $Q_{m}\left(T_{1}, \cdots, T_{k}\right)$, we have

$$
\operatorname{Sup}\left\{\left|Q_{m}\left(T_{1}, \cdots, T_{k}\right) f(y)\right|: y \in K\right\} \leqq \beta_{0} k \alpha^{m-1} \operatorname{Sup}\{|f(x)|: x \in \sigma(K)\} .
$$

If we set $S_{n}=\sum_{m=1}^{n}(-1)^{m-1} Q_{m}\left(T_{1}, \cdots, T_{k}\right)$, then for any $x \in K$ and any integers $q \geqq p \geqq 1$,

$$
\left|S_{q} f(x)-S_{p} f(x)\right| \leqq \sum_{m=p+1}^{q} \beta_{0} k \alpha^{m-1} \operatorname{Sup}\{|f(z)|: z \in \sigma(K)\}, \quad f \in C(X) .
$$

Since $\alpha<1,\left\{S_{n} f: n \geqq 1\right\}$ converges in $J_{u}$ to a $g \in C(X)$, as in the proof of Theorem 4.

For each $i, 1 \leqq i \leqq k$, we write $Q_{m}=Q_{m}^{i}+R_{m}^{i}$, where $Q_{m}^{i}$ is the sum of those terms in $Q_{m}$ which have $T_{i}$ as their left hand factor, and $R_{m}^{i}$ is the sum of the remaining terms. We will show by induction on $n$ that for $n \geqq 1$,

$$
S_{n} f=f+(-1)^{n-1} R_{n}^{i} f \mid A_{i}, \quad f \in C(X) .
$$


The case $n=1$ is obviously valid. Suppose (12) holds for some $n \geqq 1$. Since $T_{i} g=g \mid A_{i}$, we have $Q_{n+1}^{i} f=R_{n}^{i} f \mid A_{i}$, and

$$
\begin{aligned}
S_{n+1} f & =S_{n} f+(-1)^{n} Q_{n+1} f \\
& =f+(-1)^{n-1} R_{n}^{i} f+(-1)^{n} Q_{n+1}^{i} f+(-1)^{n} R_{n+1}^{i} f \mid A_{i} \\
& =f+(-1)^{n} R_{n+1}^{i} f \mid A_{i} .
\end{aligned}
$$

Hence, (12) holds for all $n \geqq 1$. Since there are fewer than $k(k-1)^{n-1}$ terms in $R_{n}^{i}$, it follows from (12) and (11) with $K=\{x\}$ that if $x \in A_{i}$ and $f \in C(X)$, then

$$
\left|S_{n} f(x)-f(x)\right|=\left|R_{n}^{i} f(x)\right|<\beta_{0} k \alpha^{n-1} \operatorname{Sup}\{|f(y)|: y \in \sigma(\{x\})\} .
$$

Therefore, $S_{n} f(x)$ converges to $f(x)$, and the proof is complete.

COROLlaRY 1. With the hypotheses of Theorem 5 , if $X$ is completely regular, and $A_{i}=\pi_{i}(X)$, then $\pi_{T}(X)=\bigcup_{i=1}^{k} \pi_{i}(X)$.

Proof. Let $B_{i}$ be a $\pi$-set of $X$ relative to $T_{i}$ for $i=1, \cdots, k$, and suppose $f=g \mid \bigcup_{i=1}^{k} B_{i}$ for some $f, g \in C(X)$. Then $Q_{m} f=Q_{m} g$ for all $m \geqq 1$, and $T f=T g$, whence $\bigcup_{i=1}^{k} B_{i}$ is a $\pi$-set of $X$ relative to $T$, and $\pi_{T}(X) \subset \bigcup_{i=1}^{k} \pi_{i}(X)=A$. This relation also holds for the series in Theorem 4. Now suppose $B$ is a $\pi$-set of $X$ relative to $T$, and $A-B \neq \varnothing$. Then we can find $f, g \in C(X)$ satisfying $f=g \mid B$ and $f \neq g \mid A$, so that $T f=T g$. But since $T f=f \mid A$ and $T g=g \mid A$, we have $T f \neq T g \mid A$, which is impossible. Hence, $B \supset A, \pi_{T}(X) \supset A$, and $\pi_{T}(X)=A$.

CoROllary 2. With the hypotheses of Theorem 5 , if $X$ is completely regular, and $A_{i}=\pi_{i}(X)$ is $a \pi$-set of $X$ relative to $T_{i}$ for $i=1, \cdots, k$, then $T^{2}=T$.

Proof. It follows from Corollary 1 and its proof that $\pi_{T}(X)$ is a $\pi$-set of $X$ relative to $T$. Since $T f=f \mid \pi_{T}(X)$, we have $T^{2}=T$. Similarly, we note that $T_{i}^{2}=T_{i}$ for $1 \leqq i \leqq k$.

The hypotheses of Theorem 5 and its corollaries can easily be satisfied when $X=E_{n}, A_{1}, \cdots, A_{k}$ are closed, pairwise disjoint $n$-balls, and $T_{i}$ is the mapping defined in Example 7 with respect to $A_{i}$ and $\phi_{i}$ for $i=1, \cdots, k$. Then we must have

$$
\left\{x:\left|T_{i} u(x)\right|<1 /(k-1)\right\} \supset \bigcup\left\{A_{j}: 1 \leqq j \leqq k, j \neq i\right\}, \quad 1 \leqq i \leqq k,
$$

that is, $\left|\phi_{i}(t)\right|<1 /(k-1)$ for a suitable set of $t$.

7. Continuous transformations. Let $X$ and $Y$ be topological spaces, and $T$ an arbitrary mapping of $C(X)$ into $C(Y)$. We will assume again in this section that $Y$ is a Hausdorff space. If $K$ is a compact subset of $Y$, we set

$$
\tau_{T}(K)=\inf \{\beta: \stackrel{\beta}{\rho}(K) \text { is defined }\}
$$

whenever the set on the right side of (13) is not empty; otherwise, we leave $\tau_{T}(K)$ undefined. The $\rho$-sets of $K$ relative to $T$ and each $\beta$ for which $\rho_{T}^{\beta}(K)$ 
is defined are precisely the $\pi$-sets of $K$ relative to $T$, as shown in Lemma 6 . If $A$ is any such set, then $A$ is evidently a $\rho$-set of $K$ relative to $T$ and $\gamma=\tau_{T}(K)$, so that $\rho_{T}^{\gamma}(K)$ is also defined.

Lemma 8. Let $T$ map $C(X)$ into $C(Y)$, and

$$
H=\left\{y \in Y: \tau_{T}(\{y\}) \text { is defined }\right\} .
$$

Then $\tau_{T}$ is lower semi-continuous on the points of $H$ with the relative topology of $Y$.

Proof. Let $y \in H, \gamma=\tau_{T}(y)$, where the latter is short for $\tau_{T}(\{y\}), \epsilon>0$, and $U=\left\{t \in E_{1}: \gamma-\epsilon<t\right\}$. Suppose that, for every neighborhood $V$ of $y$, $\left\{\tau_{T}(z): z \in V\right\} \leftarrow U$. From (13) and (6) it follows that there are $f, g \in C(X)$ satisfying

$$
\alpha=(\gamma-\epsilon / 2) \operatorname{Sup}\{|f(x)-g(x)|: x \in X\}<|T f(y)-T g(y)| .
$$

Now let $W=\{z \in Y: \alpha<|T f(z)-T g(z)|\}$. Then $W$ is a neighborhood of $y$, and we can find a $w \in W \cap H$ such that $\tau_{T}(w) \leqq \gamma-\epsilon$. But this implies

$$
\alpha>(\gamma-\epsilon) \operatorname{Sup}\{|f(x)-g(x)|: x \in X\} \geqq|T f(w)-T g(w)|,
$$

which is a contradiction. Hence, there is a neighborhood $V$ of $y$ with the property that $\left\{\tau_{T}(z): z \in V\right\} \subset U$, and the proof is complete.

Theorem 6. Let $X$ be normal, $Y$ locally compact and Hausdorff, and $T$ a mapping of $C(X)$ onto $C(Y)$ such that each compact $K \subset Y$ has a compact $\rho$-set relative to $T$ and some $\beta$, and with the property that, for each $f, g, h \in C(X)$ and each $y \in Y$,

$$
\begin{aligned}
T f(y) & =T g(y) \text { implies } \\
T(f+h)(y) & =T(g+h)(y) \text { and } T(f h)(y)=T(g h)(y) .
\end{aligned}
$$

Then there is a homeomorphism $\phi$ mapping $Y$ onto a subset of $X$ and a continuous mapping $\omega$ of $E_{1} \times Y$ (in its product topology) onto $E_{1}$ such that, for each $y \in Y, \omega \mid E_{1} \times\{y\}$ is a homeomorphism onto $E_{1}$ which satisfies a Lipschitz condition. These mappings satisfy the relation

$$
T f(y)=\omega(f(\phi(y)), y), \quad f \in C(X), y \in Y .
$$

If $T$ is one-to-one, then $\phi(Y)$ is dense in $X$.

Proof. From (14) and Theorem 2, we infer that $\mu_{T}(y)=\pi_{T}(y)$, where $\mu_{T}(y)$ is short for $\mu_{T}(\{y\})$ and similarly for $\pi_{T}(y)$, and from Lemma 7 that $\pi_{T}(y)$ is a $\pi$-set, as well as a $\rho$-set, of $\{y\}$ relative to $T$ and $\tau_{T}(y)$ for all $y \in Y$. Clearly $\pi_{T}(y) \neq \varnothing$, for otherwise $T f(y)=T g(y)$ for all $f, g \in C(X)$, and $T$ would not be onto. Suppose $\pi_{T}(y)$ contains at least two points $x_{1}$ and $x_{2}$. We construct $f_{i} \in C(X)$ satisfying $f_{i}\left(x_{j}\right)=\delta_{i j}(i, j=1,2)$. Then the mapping $\xi_{i}$ of $E_{1}$ into itself defined by $\xi_{i}(\lambda)=T\left(\lambda f_{i}\right)(y)$ is continuous. For we have 


$$
\begin{aligned}
& \tau_{T}(y)\left|\lambda-\lambda^{\prime}\right| \operatorname{Sup}\left\{\left|f_{i}(x)\right|: x \in \pi_{T}(y)\right\} \\
& \quad=\tau_{T}(y) \operatorname{Sup}\left\{\left|\lambda f_{i}(x)-\lambda^{\prime} f_{i}(x)\right|: x \in \pi_{T}(y)\right\} \\
& \quad \geqq\left|T\left(\lambda f_{i}\right)(y)-T\left(\lambda^{\prime} f_{i}\right)(y)\right|=\left|\xi_{i}(\lambda)-\xi_{i}\left(\lambda^{\prime}\right)\right|,
\end{aligned}
$$

and the first term approaches 0 as $\lambda$ tends to $\lambda^{\prime}$, since $\pi_{T}(y)$ is compact. If $\lambda \neq \lambda^{\prime}$, then $\lambda f_{i} \neq \lambda^{\prime} f_{i} \mid \pi_{T}(y)$, and $\xi_{i}(\lambda) \neq \xi_{i}\left(\lambda^{\prime}\right)$, since $\mu_{T}(y)=\pi_{T}(y)$. Thus $\xi_{i}$ is one-to-one. From $\xi_{1}(0)=\xi_{2}(0)$, we infer that $\xi_{1}\left(E_{1}\right) \cap \xi_{2}\left(E_{1}\right) \neq \varnothing$, and this set must contain more than one point, so that we can find $\lambda_{1}, \lambda_{2} \in E_{1}$ with the property that $\lambda_{1} \lambda_{2} \neq 0$ and $\xi_{1}\left(\lambda_{1}\right)=\xi_{2}\left(\lambda_{2}\right)$. Then $\lambda_{1} f_{1}=\lambda_{2} f_{2} \mid \pi_{T}(y)$, while $\lambda_{1} f_{1}\left(x_{1}\right)$ $=\lambda_{1}$ and $\lambda_{2} f_{2}\left(x_{1}\right)=0$, which is impossible. Hence, $\pi_{T}(y)$ consists of exactly one point which we denote by $\phi(y)$. The correspondence $\phi$ is one-to-one. For if $y_{1} \neq y_{2}$, we can find $f, g \in C(X)$ satisfying $T f\left(y_{1}\right)=T g\left(y_{1}\right)$ and $T f\left(y_{2}\right) \neq T g\left(y_{2}\right)$, whence $f\left(\phi\left(y_{1}\right)\right)=g\left(\phi\left(y_{1}\right)\right)$ and $f\left(\phi\left(y_{2}\right)\right) \neq g\left(\phi\left(y_{2}\right)\right)$, so that $\phi\left(y_{1}\right) \neq \phi\left(y_{2}\right)$. Let $f, g \in C(X), f^{\prime}, g^{\prime} \in C(Y), T f=f^{\prime}$, and $T g=g^{\prime}$. Then for all $y \in Y, y \in Z\left(f^{\prime}-g^{\prime}\right)$ if, and only if, $\phi(y) \in Z(f-g)$, so that $\phi\left(Z\left(f^{\prime}-g^{\prime}\right)\right)=Z(f-g) \cap \phi(Y)$. Since $\{X-Z(f-g): f, g \in C(X)\}$ is a base for the topology of $X$, and the same is true for $Y$, it follows that $\phi$ is a homeomorphism. If $(\phi(Y))^{-} \neq X$, then we can find $f, g \in C(X)$ satisfying $f=g \mid(\phi(Y))^{-}$and $f \neq g$, whence $T f=T g$. Consequently, if $T$ is one-to-one, then $(\phi(Y))^{-}=X$.

We now define the mapping $\omega$. For each $t \in E_{1}$ and $y \in Y$, we construct an $f \in C(X)$ such that $f(\phi(y))=t$ and set $\omega(t, y)=T f(y)$. This value is clearly independent of $f$ as long as $f(\phi(y))=t$, and we may take $f=t u$. If $s \neq t$, then $s u(\phi(y)) \neq t u(\phi(y))$, and $\omega(s, y) \neq \omega(t, y)$. For any $t \in E_{1}$, we can find $f \in C(X)$ satisfying $T f=t u$, so that $\omega(s, y)=t$, where $s=f(\phi(y))$. Hence, $\omega \mid E_{1} \times\{y\}$ is a one-to-one mapping onto $E_{1}$. From $\omega(t, y)=T(t u)(y)$, we obtain, by analogy with (16),

$$
\tau_{T}(y)|s-\ell|=\tau_{T}(y)|s-\ell| \cdot|u(\phi(y))| \geqq|\omega(s, y)-\omega(t, y)| \cdot
$$

Thus $\omega \mid E_{1} \times\{y\}$ satisfies a Lipschitz condition, is continuous and, therefore, a homeomorphism. In addition, $\omega \mid\{t\} \times Y$ is evidently continuous for each $t \in E_{1}$. To show that $\omega$ is continuous in the product topology, let $\epsilon>0$ and $(t, y) \in E_{1} \times Y$ be given. Then we can find a neighborhood $U$ of $y$ with the property that $\bar{U}$ is compact, and $z \in U$ implies $|\omega(t, z)-\omega(t, y)|<\epsilon / 2$. By hypothesis, $\tau_{T}(\bar{U})$ is defined and satisfies $\tau_{T}(\bar{U}) \geqq \tau_{T}(z)$ for each $z \in \bar{U}$. Clearly $\tau_{T}(z)>0$, for otherwise $T f(z)=T g(z)$ for all $f, g \in C(X)$. If $|s-t|<\epsilon /\left(2 \tau_{T}(\bar{U})\right)$ and $z \in U$, then from (17),

$$
\begin{aligned}
|\omega(s, z)-\omega(t, y)| & \leqq|\omega(s, z)-\omega(t, z)|+|\omega(t, z)-\omega(t, y)| \\
& <\tau_{T}(z) \epsilon /\left(2 \tau_{T}(\bar{U})\right)+\epsilon / 2 \leqq \epsilon,
\end{aligned}
$$

and the continuity of $\omega$ is established. Finally, (15) is a restatement of the definition of $\omega$ and $\phi$.

Corollary 1. With the same hypotheses as in Theorem 6, Tis continuous in the topology $J_{u}$. 
Proof. If the compact set $K \subset Y, \epsilon>0$, and $f \in C(X)$ are given, then from $\tau_{T}(K) \operatorname{Sup}\left\{|f(x)-g(x)|: x \in \pi_{T}(K)\right\} \geqq \operatorname{Sup}\{|T f(y)-T g(y)|: y \in K\}$

we infer that if $g$ lies in a uniform $\epsilon / \tau_{T}(K)$-neighborhood of $f$ on the compact set $\pi_{T}(K)$, then $T g$ lies in the uniform $\epsilon$-neighborhood of $T f$ on $K$.

Corollary 2. Suppose $X$ and $Y$ are compact Hausdorff spaces, and $T$ is a one-to-one mapping of $C(X)$ onto $C(Y)$ satisfying (14) and such that $\tau_{T}(Y)$ is defined. Then $X$ and $Y$ are homeomorphic.

Proof. Since $\tau_{T}(Y)$ is defined, $X$ is a compact $\rho$-set of every compact $K \subset Y$ relative to $T$ and $\tau_{T}(Y)$. From Theorem $6, \phi(Y)$ is compact and dense in $X$, so that $\phi(Y)=X$.

The last result is related to a theorem of Stone [4] that if $X$ and $Y$ are compact, and $T$ is an isometry in the sup norm between $C(X)$ and $C(Y)$, then $X$ and $Y$ are homeomorphic. The condition that $T$ be an isometry implies, by a theorem of Banach, that $T$ is a linear transformation followed by a translation, and this is easily seen to imply the first half of (14). For other related results $\mathrm{cf} .[1, \mathrm{p} .23]$.

We recall that a subset $A$ of a topological space $X$ is said to be $C$-embedded in $X$ if every continuous, real-valued function on $A$ has a continuous extension to $X$.

TheOREM 7. Let $X$ and $Y$ be arbitrary spaces, $\phi$ a homeomorphism of $Y$ onto $a C$-embedded subset of $X$, and $\omega$ a continuous mapping of $E_{1} \times Y$ (in the product topology) onto $E_{1}$ such that, for each $y \in Y, \omega \mid E_{1} \times\{y\}$ is a homeomorphism onto $E_{1}$. If $T$ is defined as in (15), then $T$ maps $C(X)$ onto $C(Y)$ and satisfies (14). If $\phi(Y)$ is dense in $X$, then $T$ is one-to-one. If $X$ is completely regular, and $D$ is a closed subset of $Y$, then $\mu_{T}(D)=\pi_{T}(D)=(\phi(D))^{-}$, and $(\phi(D))^{-}$is a $\pi$-set of $D$ relative to $T$. If $X$ is Hausdorff, $K \subset Y$ is compact, and there is a $\beta \geqq 0$ satisfying

$$
\beta|s-t| \geqq|\omega(s, y)-\omega(t, y)|, \quad s, t \in E_{1}, y \in K,
$$

then $K$ has a compact $\rho$-set relative to $T$ and $\beta$.

Proof. For any $f \in C(X)$, the continuity of $T f$ on $Y$ follows from that of $\phi$ and $\omega$. If $g \in C(Y)$ is given, we define $f$ on $\phi(Y)$ by the relation $\omega(f(\phi(y)), y)$ $=g(y)$. Since $\omega \mid E_{1} \times\{y\}$ is a homeomorphism onto $E_{1}, f$ is single-valued on $\phi(Y)$. Suppose $f$ were discontinuous at the point $\phi(y)$. Then we can find $\epsilon>0$ with the property that, for every neighborhood $U$ of $y$, there is a $z \in U$ satisfying $|f(\phi(z))-f(\phi(y))| \geqq \epsilon$. In fact, either (i) every neighborhood $U$ of $y$ contains a $z$ satisfying $f(\phi(z))-f(\phi(y)) \geqq \epsilon$, or (ii) every neighborhood contains a $z$ satisfying $f(\phi(z))-f(\phi(y)) \leqq-\epsilon$. There is also a neighborhood $V$ of $y$ with the property that, for each $z \in V, \omega \mid E_{1} \times\{z\}$ is an increasing or decreasing function according as $\omega \mid E_{1} \times\{y\}$ is increasing or decreasing. For suppose, to be definite, that $\omega \mid E_{1} \times\{y\}$ is increasing. Choose $s, t \in E_{1}, s<t$, and a 
neighborhood $V \subset Y$ of $y$ such that $z \in V$ implies $|\omega(s, z)-\omega(s, y)|<\delta / 2$ and $|\omega(t, z)-\omega(t, y)|<\delta / 2$, where $\delta=\omega(t, y)-\omega(s, y)$. Then

$$
\begin{aligned}
\omega(t, z)-\omega(s, z)= & \omega(t, z)-\omega(t, y)+\omega(t, y)-\omega(s, y) \\
& +\omega(s, y)-\omega(s, z)>-\delta / 2+\delta-\delta / 2=0,
\end{aligned}
$$

so that $\omega \mid E_{1} \times\{z\}$ is increasing. A similar argument applies if $\omega \mid E_{1} \times\{y\}$ is decreasing. Returning to the continuity of $f$, we will assume first that case (i) above holds, and $\omega \mid E_{1} \times\{y\}$ is increasing. There is then a neighborhood $W$ of $y$ such that $W \subset V$, and $z \in W$ implies $|g(z)-g(y)|<\alpha / 2$ and

$$
|\omega(f(\phi(y))+\epsilon, z)-\omega(f(\phi(y))+\epsilon, y)|<\alpha / 2,
$$

where

$$
\alpha=|\omega(f(\phi(y))+\epsilon, y)-\omega(f(\phi(y)), y)| .
$$

If we choose $z$ so as to satisfy (i) with $U=W$, then

$$
\begin{aligned}
\omega(f(\phi(y))+\epsilon, y)-\omega( & f(\phi(y))+\epsilon, z) \geqq \omega(f(\phi(y))+\epsilon, y)-\omega(f(\phi(z)), z) \\
& =\omega(f(\phi(y))+\epsilon, y)-\omega(f(\phi(y)), y)+g(y)-g(z) \\
& >\alpha-\alpha / 2=\alpha / 2,
\end{aligned}
$$

which contradicts (19). If $\omega \mid E_{1} \times\{y\}$ is increasing, and (ii) holds instead of (i), the same reasoning applies if we replace $+\epsilon$ by $-\epsilon$ in (19), (20), (21), reverse the inequality signs in (21), and change the last part to read $<-\alpha$ $+\alpha / 2=-\alpha / 2$. If $\omega \mid E_{1} \times\{y\}$ is decreasing, a similar argument leads to contradiction. Hence, $f$ is continuous at $\phi(y)$. Since $\phi(Y)$ is $C$-embedded in $X$, we can extend $f$ to an element of $C(X)$ which $T$ maps into $g$. Therefore, $T$ is onto.

It is easily seen that, for every $y \in Y$ and $f, g \in C(X), f(\phi(y))=g(\phi(y))$ if, and only if, $T f(y)=T g(y)$. Thus from $T f(y)=T g(y)$ we obtain $(f+h)(\phi(y))$ $=(g+h)(\phi(y)), \quad(f h)(\phi(y))=(g h)(\phi(y)), \quad T(f+h)(y)=T(g+h)(y), \quad T(f h)(y)$ $=T(g h)(y)$, and (14) is verified. If $\phi(Y)$ is dense in $X, f, g \in C(X)$, and $f \neq g$, then $f \neq g \mid \phi(Y)$, and $T f \neq T g$, whence $T$ is one-to-one. For any closed $D \subset Y$, we have $\mu_{T}(D) \supset(\phi(D))^{-}$, and $(\phi(D))^{-}$is a $\pi$-set of $D$ relative to $T$. If $X$ is completely regular, we infer from (5) that $\mu_{T}(D)=\pi_{T}(D)=(\phi(D))^{-}$. Finally, if $K \subset Y$ is compact, (18) is satisfied, and $f, g \in C(X)$, then

$$
\beta|f(\phi(y))-g(\phi(y))| \geqq|T f(y)-T g(y)|, \quad y \in K,
$$

and $\phi(K)$ is a compact $\rho$-set of $K$ relative to $T$ and $\beta$.

One of the simplest examples of the function $\omega$ is obtained by choosing $f_{0}, f_{1} \in C(Y)$ with $Z\left(f_{1}\right)=\varnothing$ and setting $\omega(t, y)=f_{0}(y)+t f_{1}(y)$. When $f_{0}=\theta$ and $f_{1}=u$, we have

$$
T f(y)=f(\phi(y)), \quad y \in Y, f \in C(X) .
$$


For this choice of $\omega, T$ evidently becomes a homomorphism of the ring $C(X)$ into the ring $C(Y)$. From Theorem 7 we obtain the known results that if $\phi(Y)$ is $C$-embedded in $X$, then $T$ is onto, and if $\phi(Y)$ is also dense in $X$, then $T$ is an isomorphism. The relation (22) between $T$ and $\phi$ has itself been studied, and a strong duality between homomorphisms of $C(X)$ into $C(Y)$ and continuous mappings of $Y$ into $X$ has been revealed (cf. [1, Chapter 10]). If we make the additional assumption in Theorem 6 that $T$ is a ring homomorphism (which implies (14)), then $\omega(t, y)=t$ for each $t \in E_{1}$ and $y \in Y$. Indeed, from (15) we have

$$
\begin{aligned}
\omega(s+t, y) & =\omega((s u+t u)(\phi(y)), y)=T(s u+t u)(y) \\
& =T(s u)(y)+T(t u)(y)=\omega(s, y)+\omega(t, y),
\end{aligned}
$$

and similarly $\omega(s t, y)=\omega(s, y) \omega(t, y)$. Thus $\omega \mid E_{1} \times\{y\}$ is a ring homomorphism of $E_{1}$ onto itself which must be the identity mapping. Hence, $T$ satisfies (22). This result is related to a known theorem [1, p. 142] that if $X$ is completely regular, $Y$ realcompact $[1$, p. 114], and $T$ is a homomorphism of $C(X)$ into $C(Y)$ such that $T u=u$, then there is a unique continuous mapping $\phi$ of $Y$ into $X$ such that (22) is satisfied.

\section{REFERENCES} 1960.

1. L. Gillman and M. Jerison, Rings of continuous functions, Princeton, Van Nostrand,

2. E. Hewitt, On two problems of Urysohn, Ann. of Math. (2) vol. 47 (1946) pp. 503-509.

3. J. L. Kelley, General topology, New York, Van Nostrand, 1955.

4. M. H. Stone, Applications of the theory of Boolean rings to general topology, Trans. Amer. Math. Soc. vol. 41 (1937) pp. 375-481.

University of British Columbia, Vancouver, British Columbia 